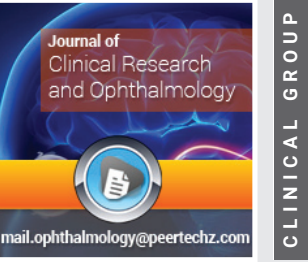

\title{
Congruous homonymous hemianopia due to occipital lobe infarction
}

\author{
K Suresh ${ }^{1 *}$, Arpita $^{2}$ and Sujitkumar ${ }^{3}$ \\ Karnataka, India \\ ${ }^{2}$ Consultant Retina, Narayan Netralaya, Bengaluru, India \\ ${ }^{3}$ Neuro-Physician, Apollo Hospital, Seshadripuram, Bengaluru, India
}

${ }^{1} \mathrm{MD}$, DIH, FIAP, FIPHA, FISCD, Public Health Consultant \& Visiting Professor, MPH, KSRDPRU, Gadag,

Received: 17 December, 2020

Accepted: 23 December, 2020

Published: 24 December, 2020

*Corresponding authors: K Suresh, MD, DIH, FIAP, FIPHA, FISCD, Public Health Consultant \& Visiting Professor, MPH, KSRDPRU, Gadag, Karnataka, India, E-mail:ksuresh.20@gmail.com

Keywords: Brain; Chiasm; Contralateral vision; Homonymous Hemianopsia (HH); Lesion; MRI; Neurological adversities; Occipital lobes; Optics; Retrochiasmal visual pathway; Sight; Stroke; Trauma; Vision

https://www. peertechz.com

Check for updates

\section{Summary}

Optic disorder in which the visual field defects in both eyes are completely symmetric in extent and intensity are defined as Congruous homonymous hemianopia. Here is such a case that I followed early this year. A sixty- five years old male, diabetic since 1994, Hypertensive for 14 years and ischemic heart disease for 10 years under ayurvedic treatment reported with a history of fall from the bed while sleeping in the midnight $11^{\text {th }} \& 12^{\text {th }}$ February 2020 and found himself fully blurred bilateral vision with severe headache on the morning of $15^{\text {th }}$. After a local consultation and laser therapy attempt did not benefit, he landed in Bangalore. Suspecting diabetic Retinopathy, we took him to Narayana Netralaya a super-specialty eye hospital. Detailed examination by a retina specialist confirmed right homonymous hemianopia with no significant Retinal findings to explain the defective vision. An MRI Brain and Orbit plain and contrast was ordered, that revealed Subacute Ischemic Infarct in Bilateral PCA territory. Other investigations did indicate uncontrolled diabetes hypertension and evidence of Ischemic Heart Diseases S/P PTCA. The case was referred to a neuro-physician who put him on Inj. Perfalgan 1 G IV SOS, Inj. PAN-40 Mg IV once a day x3, Inj. Emeset 4 mg IV SOS, Tab Clopilet A 150 mg once a day in the afternoon and other supportive treatment. There was an improvement in the vision within 24 hours as he could count fingers at 1 foot. Hospital stay lasted for 3 days and the vision was improving day by day. The second follow-up was uneventful but before a bit delayed third follow-up the patient died of cardiac arrest within 2 months of the first episode.

\section{Background}

The vision can be affected from a myriad of local ophthalmologic conditions and those affecting the neural structures conveying visual information from retina to brain. Such neuro-ophthalmologic diseases can be due to damage at any location from optic disc, optic nerve, optic chiasm, optic tract to optic radiation and occipital cortex. Various causes of neurogenic vision loss include - Optic neuropathy (demyelinating, ischemic, toxic, infectious, secondary to chronically raised Intracranial Pressure (ICP)), compression of the visual pathway because of space occupying lesions and tubercular arachnoiditis in optochiasmatic region, ischemic involvement of the post-chiasmatic visual pathway as in case of stroke, Cerebral Venous Thrombosis (CVT), cortical vision loss due to various etiologies such as stroke, Posterior Reversible Encephalopathy Syndrome (PRES), Subacute Sclerosing Panencephalitis (SSPE). I heard of the entity called 'Congruous homonymous hemianopia' first time in my 52 years of public health experience. Initially the condition misled us to think of a serious ophthalmic problem like diabetic retinopathy as the patient was a known diabetic for over 25 years or other eye related nerve problem and seek care in an ophthalmic superspeciality hospital. The outcome was also a typical text- book description of the condition that ended in the death of the individual in about a month's time despite showing signs of improvement in visual acuity. The literature review indicated extreme rarity of such conditions worldwide.

\section{Case presentation}

A cousin of mine, a male of sixty-five years known Diabetic for over 26 years, Hypertensive for last 15 years, and under treatment for Ischaemic heart disease for last 4 years landed on the morning of 18 February 2020 in Bengaluru seeking my professional support. His main complaint was sudden bilateral blindness following a fall from a bed in the midnight of 11/2/20. After a private ophthalmologist in a small town examined and on the morning of $12 / 2 / 20$, recorded vision RE- Finger count 
at 0.5 meters and LE Finger count at 1 meter and referred to a well-equipped eye hospital in Hubballi $100 \mathrm{kms}$ away. In the second hospital a laser therapy was tried on 15/2/20 with no improvement in the vision. They were advised for a second laser therapy session with no assurance of a positive outcome. Since they did not appear convincing in their approach and diagnosis, the family came to Bengaluru on the morning of 18 February 2020

We took him to Narayan Netralaya a popular super speciality eye hospital. The case was assigned to a Retina specialist, who after a careful examination ordered for Orbit plain and contrast, that did not indicate any retinal findings to explain the near complete blindness. The Retina specialist suspected right homonymous hemianopia and took a neurologist's opinion who ordered for an MRI of the Brain, that revealed Subacute Ischemic Infarct in Bilateral PCA territory.

The case was admitted under the supervision of a neurologist who managed with Inj. Perfalgan 1 G IV SOS, Inj. PAN-40 Mg IV once a day x3, Inj. Emeset $4 \mathrm{mg}$ IV SOS, Tab Clopilet A $150 \mathrm{mg}$ once a day in the afternoon and other supportive treatment. There was an improvement in the vision within 24 hours, as he could count fingers at 1 foot. Hospital stay lasted for 3 days and the vision was improving day by day and was able to recognize the faces of family members by then and walk around without any support though the clarity was around $50 \%$. He was discharged on the $4^{\text {th }}$ day with continued medication and follow-up after a fortnight on 2 March 2020.

\section{Investigations done}

At Narayan Netralaya: a) fundoscopy b) Orbit plain and Contrast b) Hb1AC -9.2, Urine Glucose 1+ Proteins 1+, hypertension and ECG for the evidence of Ischemic Heart Diseases -S/P PTCA.

At Focus Diagnostic Centre- a) Plain MRA of Brain both orbits- Indicated irregular lesions in bilateral occipital lobes- suggestive of posterior reversible encephalopathy involving bilateral occipital lobes, causing both ischaemic and haemorrhagic lesions. b) Plain MRA of Circle of Willis- No evidence of any sclerotic or occlusive vascular disease. c) Plain MRA of Neck vessels- No significant abnormalities.

\section{Outcome \& Follow-up}

The second follow-up on $2^{\text {nd }}$ March was uneventful and the Neurologist was also fully satisfied with the progress. The patient was able to walk on his own with near clear vision, BP and Blood sugar were within acceptable range. He was advised a further medication for 4 weeks. The medications included i) Caps Clopitab (combination of two Aspirin and Clopidogrel -antiplatelet medicines) $150 \mathrm{mg}$ once a day in the afternoon for 4 weeks ii) Tab Rosuvas $20 \mathrm{mg}$, (to reduce bad cholesterol) one tablet every night iii) Tab Strocit (Citicoline) $500 \mathrm{mg}$ Twice a day iv) Tab Losakind $50 \mathrm{mg}$ (for controlling Hypertension) once in the morning along $\mathrm{v}$ ) Tetrafol plus (L METHYLFOLATE2.8MG + PYRIDOXAL PHOSPHATE-25MG+MECOBALAMIN$2 \mathrm{MG}$ ) once a day with his routine antidiabetic drugs.
Due to the Corona Pandemic the follow up visit was delayed a bit. A final follow-up was planned for $15^{\text {th }}$ April.

On $14^{\text {th }}$ April 2020 he had a sudden discomfort when he went for an early evening walk and died of Cardiac arrest before reaching the nearest hospital.

\section{Discussion}

\section{Causes of sudden blindness}

Migraines are the most common causes of temporary vision loss. They cause blind spots, or one may feel like one is seeing flashing lights. Migraines cause Sudden Blindness in both eyes. Giant cell arteritis is another cause of temporary blindness in one eye. This can cause temporary vision loss in people who are over 50 years old. If left untreated, it can cause long-term or permanent blindness too. Another cause of temporary vision loss is retinal vasospasm in which blood flow is impacted due to the tightening of the blood vessels in your retina.

Closed-angle glaucoma can lead to temporary blindness in one eye due to excessive pressure on the eye, if not diagnosed and treated on time, may lead to permanent vision loss as well. Another cause of Sudden Blindness is vitreous haemorrhage in which blood leakage occurs. Rare causes of sudden loss of vision include a retinal migraine in one eye. About $10 \%$ of people who suffer from epilepsy, the seizures can have an impact on the occipital lobe leading to loss of vision when the seizure is taking place or after the seizure. Another rare cause of Sudden Blindness is Uhthoff phenomenon which affects people who have multiple sclerosis. Retinal vein occlusion rarely causes Sudden Blindness, a temporary vision loss. Sudden total vision loss can be caused due to a clot. A formation of a clot can cause temporary vision loss which can lead to a permanent or total loss of vision when prompt treatment is not undertaken. The clot blocks the retinal artery which may cause blindness or when there is blockage due to a clot in a blood vessel [1].

In a study with a prevalence of visual impairment of $24.5 \%$ (95\% CI: $20.9 \%-28.1 \%$ ), Cataract was the leading cause of visual impairment (50.7\%), followed by uncorrected refractive error $(36.8 \%)$. Despite a reportedly high CSR, cataract remains the predominant cause of blindness [2].

In another study where a total of 900 eyes were examined. Visual impairment and blindness were, seen in 135 (30\%) and $36(8 \%)$ individuals, respectively. The most common cause of blindness was cataract, followed by corneal opacity, glaucoma, refractive error, diabetic retinopathy, macular scar, age related macular degeneration, retinal detachment, retinitis pigmentosa. There is an increase in blindness and visual impairment due to corneal diseases and glaucoma which was not seen a decade ago. The availability and accessibility to eye care particularly for corneal diseases and glaucoma need augmentation in Northern India [3]

In a study of Neurogenic vision loss of 64 patients evaluated, 54 cases were due to diseases of anterior visual pathway and rest 10 had cortical vision loss. The etiologic distribution wereIsolated optic neuritis- 12 (19\%), multiple sclerosis- $4(6.3 \%)$, 
neuromyelitis Optica- $5(7.9 \%)$, tubercular meningitis- 15 (23.8\%), non-arteritic ischemic optic neuropathy, ischemic optic neuropathy complicating cavernous sinus thrombosis, cryptococcal meningitis, malignant infiltration of optic nerve, Crouzon's syndrome, calvarial thickening and traumatic occipital gliosis- $1(1.6 \%)$ case each, idiopathic intracranial hypertension, pituitary adenoma, acute disseminated encephalomyelitis, posterior reversible leukoencephalopathy$3(4.8 \%)$ cases each, cortical venous thrombosis $5(7.9 \%)$, subacute sclerosing panencephalitis- 4 (6.3\%) cases [4].

A large portion of the central nervous system is dedicated to vision and therefore strokes have a high likelihood of involving vision in some way. Vision loss can be the most disabling residual effect after a cerebral infarction. Transient vision problems can likewise be indicator of a stroke and demand evaluation after recognition of visual symptoms that can prevent future vascular injury. Optic disorder in which the visual field defects in both eyes are completely symmetric in extent and intensity are defined as Congruous homonymous hemianopia.

The word Homonymous Hemianopsia ( $\mathrm{HH})$ is quite unfamiliar with most people. In every average human being, the left half of the occipital lobe in the brain is responsible for processing the visual information coming from the view perceived through the right eye. and the right side of the occipital lobe processes visual information coming from the view perceived by the left eye [1]. In the case of homonymous hemianopsia, there is an injury to either side of occipital lobes in the brain leads to a hindrance in the respective eye's vision. This leads to the individual's vision to be vertically segregated, thereby causing every object or view to be divided into two halves, resulting in only one half of every sight and the other half as pitch black. This defective vision occurs solely due to the damages occurring in the occipital lobes. There is no defect of any sort in either of the eyes. Stroke is the major cause of $\mathrm{HH}$, followed by trauma and tumours. Unilateral damage occurring in the retrochiasmal visual pathway leads to bilateral vision loss, affecting the contralateral visual field. The most remarkable damages occurring in the brain resulting from $\mathrm{HH}$ are lesions in the occipital lobe ( $45 \%)$, followed by damage to the optic radiations $(32 \%)$, lesions of the optic tract $(10 \%)$, lateral geniculate nucleus (LGN) $(1.3 \%)$, or a combination of several areas $(11 \%)[5]$

In this case study, we discuss first, anatomy and the vascular supply of the visual system followed by the different causes of sudden blindness, neurogenic blindness and stroke syndromes which involve vision and finally, topics involving the assessment, prognosis, treatment, and therapeutic intervention of vision-specific stroke topics.

\section{Anatomy}

The prechiasmal visual pathway consists of the axons from the nerve fibre layer of the retina, which forms the optic nerves and then travel medially towards the optic canals. The prechiasmal optic nerves are supplied by ophthalmic artery and internal carotid artery (ICA) pial vessels. The central retinal artery, a branch of the ophthalmic artery, provides the blood supply to the retina. The optic nerves conjoin to form the optic chiasm, which is supplied by Circle of Willis. The retrochiasmal visual pathway encompasses the region from the optic chiasm to the visual cortex. Fibres from the optic chiasm and the optic tracts, supplied primarily by the Anterior Choroidal Artery (AChA), travel to the Lateral Geniculate Body (LGB). Though the AChA, a branch of the Internal Carotid Artery (ICA) and Lateral Posterior Choroidal Artery (LPChA), a branch of the Posterior Cerebral Artery (PCA) provide dual vascular supply to the LGBs, the terminal anastomosis is vulnerable to ischaemia. Optic radiations originate from the Lateral Geniculate Nucleus (LGN) and are divided into superior, inferior, and central nerve fibres. The optic radiations are predominantly supplied by the posterior and middle cerebral arteries and the AChA. Inferior fibres, known as Meyer's Loop, travel to the temporal lobe, while the superior and central nerve fibre bundles travel to the parietal lobes. The termination of optic radiations is in the visual striate cortex (V1) in the occipital lobe; superior and inferior to the calcarine fissure. The occipital cortex is largely supplied by the PCAs, which are the terminal branches off the basilar artery (Figure 1). Infarctions in the PCA territories can cause cortical vision loss. Bilateral PCA infarcts as in 'top of the basilar syndrome' can result in cortical blindness.

Radiographic angiography of the posterior circulation. Bilateral vertebral arteries fuse to form the basilar artery. The ultimate branches of the basilar artery form the Posterior Cerebral Arteries (PCAs, arrows), which supply the visual cortex [2].

\section{Visual stroke syndromes}

1. The Ophthalmologist or Neurologist use knowledge of these anatomical landmarks and their corresponding blood supply to clinically localise a stroke involving vision by recognising effects of their dysfunction on the clinical examination. Monocular visual loss due to prechiasmal ischaemia: Prechiasmal vision loss can be caused by retinal ischaemia secondary to occlusion within the ophthalmic artery vascular supply. Preceding symptoms of non-visual strokes often include TMVL. Over $75 \%$ of these patients experience associated decreased visual acuity.

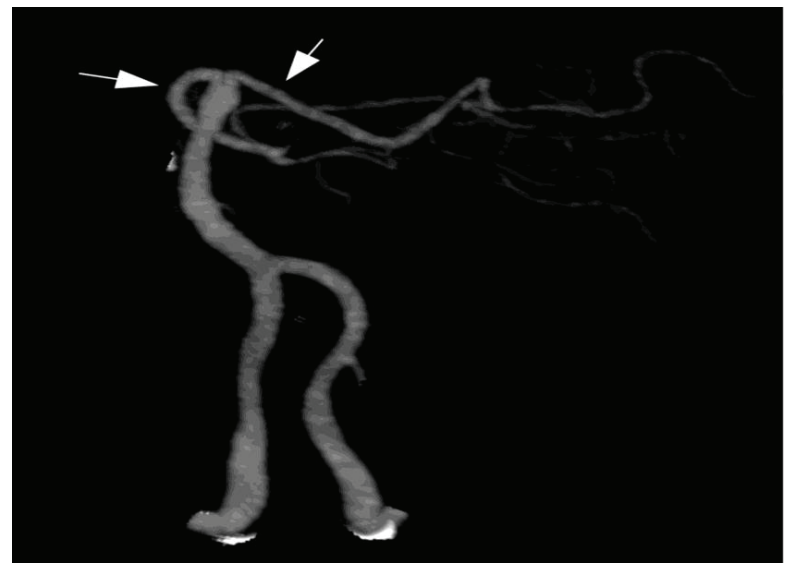

Figure 1: Terminal branches off the basilar artery

Citation: Suresh K, Arpita, Sujitkumar (2020) Congruous homonymous hemianopia due to occipital lobe infarction. J Clin Res Ophthalmol. 7(2): 105-109. 
2. Bitemporal hemianopia due to chiasmal ischaemia: Chiasmal strokes are rare, owing to the rich supply of collateral circulation provided by the Circle of Willis to the optic chiasm. When chiasmal strokes do occur, patients experience acute onset bitemporal hemianopia.

3. Homonymous Hemianopia $(\mathrm{HH})$ due to post-chiasmal ischaemia- Post-chiasmal strokes occur secondary to ischaemia in the LGB, optic radiations, or occipital lobe and can manifest as sectoranopias, quadrantanopias, or hemianopias, either congruous or incongruous.

4. Congruous homonymous hemianopia due to occipital lobe infarction -Up to $8 \%-25 \%$ of patients who had a stroke can develop visual field loss. Stroke is the most common causative factor for $\mathrm{HH}$ and correspondingly, $\mathrm{HH}$ is the most common form of visual field loss following stroke Visual disturbance induced by bilateral LGB infarction is a rare occurrence [2].

\section{The importance of congruous homonymous hemiano- pia}

Congruous homonymous hemianopia due to occipital lobe infarction is estimated to be $8 \%-25 \%$ of patients who had a stroke that can develop visual field loss. A large portion of the central nervous system is dedicated to vision and therefore strokes have a high likelihood of involving vision in some way. Vision loss can be the most disabling residual effect after a cerebral infarction. Transient vision problems can likewise be a harbinger of stroke and prompt evaluation after recognition of visual symptoms can prevent future vascular injury [6].

\section{The historical context of this condition}

German physiologist Hermann Munk (1839-1912) observed the behaviour of monkeys for up to 5 years after he made lesions in the angular gyrus or in the occipital lobes and concluded that the "critical site of vision" is in the occipital lobes. Albrecht von Graefe (1828-1870) introduced visual field examination into daily practice, when he invented campimetry (von Graefe, 1856). He also noted that lesions of the occipital cortex and temporal lobe caused homonymous hemianopsia. By the 1890 s devices were constructed to measure visual field defects accurately. Moses Allen Starr (1854-1932), a New York neurologist, confirmed Munk's observations from the study of multiple human autopsies of patients with hemianopsia, who had lesions in their occipital lobes (Starr, 1884). Gordon Holmes (1918) serially mapped visual fields of dozens of soldiers recovering from gunshot wounds sustained in their occipital lobes. He discovered that the centre for macular vision lies in the most posterior portion of the visual cortex, probably on the margins and on the lateral surfaces of the occipital lobes ... Severe lesions of the visual cortex produce complete blindness in the corresponding portions of the visual fields ... The defects of vision in the fields of the two eyes are always congruous and superimposable [7].

\section{Difficulties in diagnosing or treatment}

The most common cause of $\mathrm{HH}$ in adults is stroke. Approximately $8 \%-25 \%$ of stroke patients have permanent
$\mathrm{HH}$, and $52 \%-70 \%$ of hemianopias are caused by stroke. As the population ages and stroke patients live longer, the incidence of stroke and resultant $\mathrm{HH}$ is likely to increase $[7,8]$.

Visual fields, particularly when correlated with other symptoms, provide valuable information regarding the location of brain lesions. Goldmann perimetry is useful in detecting neurologic visual field loss. Humphrey automated perimetry is widely used for assessing visual field defects. Confrontation visual field testing is not sensitive at detecting visual field loss, but it may be the only method available. The most sensitive individual method of confrontation visual field testing is kinetic testing using a $5 \mathrm{~mm}$ red bead. This picks up $43 \%$ of mild defects and $89 \%$ of severe defects. The overall sensitivity using the kinetic red bead is $74 \%$, but this improves to $78 \%$ when combined with static finger wiggle testing [8-10]. A complete $\mathrm{HH}$ affects the entire hemifield of both eyes and can occur with a lesion anywhere posterior to chiasm and cannot be further localized based on visual field appearance alone. A congruous visual field defect is identical between the two eyes, whereas an incongruous defect differs in appearance between the eyes. For lesions behind the LGN, visual field defects are generally more congruous if the lesion is located more posteriorly along the visual pathway.

In a retrospective observational study, charts of 548 incomplete (373 congruent $\mathrm{HH}$ and 175 incongruent $\mathrm{HH}$ ) patients with $\mathrm{HH}$ over 15 years were reviewed. Stroke caused $75 \%$ of congruent $\mathrm{HH}$ and $55.8 \%$ of incongruent $\mathrm{HH}$; trauma and tumours caused $20.5 \%$ of congruent $\mathrm{HH}$ and $34.5 \%$ of incongruent $\mathrm{HH}(\mathrm{P}<.001)$. The lesion locations in congruent $\mathrm{HH}$ vs incongruent $\mathrm{HH}$ included occipital lobe in $47.9 \%$ vs $21.3 \%$, occipital lobe and optic radiations in $8.3 \%$ vs $5.6 \%$, optic radiations in $32.4 \%$ vs $50.6 \%$, optic tract in $7.2 \%$ vs $16.3 \%$, and other locations in $4.2 \%$ vs $6.3 \%(\mathrm{P}<.0001)$. Although there was a trend toward more congruent $\mathrm{HH}$ for lesions of the posterior visual pathways $(\mathrm{P}<.001), 50 \%$ of optic tract lesions and $59 \%$ of optic radiation lesions produced congruent $\mathrm{HH}$. Study inferred that lesions involving the occipital lobe produce congruent $\mathrm{HH}$, at least $50 \%$ of lesions in other locations also produced congruent $\mathrm{HH}[10,11]$.

A well conducted visual field testing and careful evaluation of the visual field defect pattern can localize the lesion site approximately along the optic pathway that can be confirmed by modern imaging modalities, reported Shikha Baisakhiya and Amit Agrawal in a case report where the lesion was localized more anteriorly where anatomically the peripheral vision function in the cerebral cortex is located, sparing of the occipital pole was responsible for macular sparing in the patient $[12,13]$

\section{Take home messages}

- Strokes could involve vision in some way as large portion of central nervous system is dedicated to vision.

- Bilateral Blindness (transient or permanent) especially among known diabetic or hypertensives could be harbinger of stroke needing prompt evaluation.

Citation: Suresh K, Arpita, Sujitkumar (2020) Congruous homonymous hemianopia due to occipital lobe infarction. J Clin Res Ophthalmol. 7(2): 105-109. 
- Post-chiasmal strokes occur secondary to ischaemia in the LGB, optic radiations, or occipital lobe and can manifest as sector anopias, quadrantanopias, or hemianopias, either congruous or incongruous.

- Homonymous Hemianopia is the most common form of visual field loss following stroke

- Congruous homonymous hemianopia due to occipital lobe infarction $-8 \%-25 \%$ of patients who had a stroke can develop visual field loss.

\section{References}

1. Sudden blindness causes. Link: https://bit.ly/2WEnrme

2. Vignesh D, Gupta N, Kalaivani M, Goswami AK, Nongkynrih B, et al. (2019) Prevalence of visual impairment and its association with vision-related quality of life among elderly persons in a resettlement colony of Delhi. J Family Med Prim Care 8: 1432-1439. Link: https://bit.ly/3aFAIDu

3. Baldev VF, Chopra R, Batra N, Singh S (2017) Pattern of Ocular Morbidity in the Elderly Population of Northern India. J Clin Diagn Res 11: NC20-NC23. Link: Link: https://bit.ly/3pjfWxp

4. Verma R, Gupta M, Chaudhari TS (2014) Neurogenic vision loss: Causes and outcome. An experience from a tertiary center in Northern India. J Neurosci Rural Pract 5: 340-348. Link: https://bit.ly/3pnBE3w
5. Santra P (2020) Amity university, Homonymous Hemianopsia: The vision in fragments Eyes and stroke. Link: https://bit.ly/3mLRIzx

6. Pula JH, Yuen CA (2017) Eyes and stroke: the visual aspects of cerebrovascular disease. Stroke Vasc Neurol 2: 210-220. Link: https://bit.ly/38AXRnE

7. Homonymous Hemianopsia. History of Neurology, Edward J Fine, M Ziad Darkhabani, in Handbook of Clinical Neurology.

8. Gilhotra JS, Mitchell P, Healey PR, Cumming RG, Currie J (2002) Homonymous visual field defects and stroke in an older population. Stroke 33: 2417-2420. Link: https://bit.ly/3pmw3KI

9. Zhang X, Kedar S, Lynn MJ, Newman NJ, Biousse V (2006) Homonymous hemianopia in stroke. J Neuroophthalmol 26: 180-183. Link: https://bit.ly/3nN6n9H

10. Lavin P, Donahue S (2008) Magnetic resonance imaging changes associated with transient homonymous hemianopia in patients with nonketotic hyperglycaemia. Arch Ophthalmol 126: 1467-1468.

11. Taban M, Naugle RI, Lee MS (2007) Transient homonymous hemianopia and positive visual phenomena in patients with nonketotic hyperglycaemia. Arch Ophthalmol 125: 845-847. Link: https://bit.ly/2M6s7iT

12. Kedar S, Zhang X, Lynn MJ, Newman NJ, Biousse V (2007) Congruency in homonymous hemianopia. Am J Ophthalmol 143: 772-780. Link: https://bit.ly/2WJj5KM

13. Baisakhiya S, Agrawal A (2011) Homonymous Hemianopia with Macular Sparing. Nigerian Journal of Ophthalmology 18: 69-70.

\section{Discover a bigger Impact and Visibility of your article publication with}

\section{Peertechz Publications}

\section{Highlights}

* Signatory publisher of ORCID

* Signatory Publisher of DORA (San Francisco Declaration on Research Assessment)

* Articles archived in worlds' renowned service providers such as Portico, CNKI, AGRIS, TDNet, Base (Bielefeld University Library), CrossRef, Scilit, J-Gate etc.

* Journals indexed in ICMJE, SHERPA/ROMEO, Google Scholar etc.

* OAI-PMH (Open Archives Initiative Protocol for Metadata Harvesting)

- Dedicated Editorial Board for every journal

* Accurate and rapid peer-review process

* Increased citations of published articles through promotions

* Reduced timeline for article publication

Submit your articles and experience a new surge in publication services (https://www.peertechz.com/submission).

Peertechz journals wishes everlasting success in your every endeavours.

Copyright: @ 2020 Suresh K, et al. This is an open-access article distributed under the terms of the Creative Commons Attribution License, which permits unrestricted use, distribution, and reproduction in any medium, provided the original author and source are credited. 